\title{
Metodologías de medición de la calidad de los servicios aplicadas a una biblioteca universitaria
}

\begin{abstract}
RESUMEN
El trabajo realizado ha permitido identificar cuatro dimensiónes relacionadas con la calidad de servicio de la Biblioteca de la Universidad Nacional Mayor de San Marcos UNMSM. Tres de ellas, las que se han denominado "Aspectos Tangibles, Empatía y Fiabilidad ", pueden considerarse equivalentes a las inicialmente obtenidas por Parasuraman, Zeithaml y Berry. La restante, es la integración de Capacidad de Respuesta y Seguridad que se ha denominado "Respuesta-Seguridad". Las dimensiónes encontradas son coincidentes con lo hallado en otras investigaciones realizadas dentro del sector educativo universitario, pero no están incluidas dentro de las encontradas por dichos autores. Asimismo, se probó el uso de las técnicas de redes neuronales y agrupación con $\mathrm{K}$ means; dando un resultado equivalente al del análisis factorial. En el caso de redes neuronales se utilizaron las de Aprendizaje Competitivo con Learning Vector Quantitative LVQ.
\end{abstract}

Palabras clave: medición de la calidad de servicios, biblioteca, análisis factorial, $\mathrm{K}$ means, redes neuronales

METHODOLOGIES FOR MEASURING THE QUALITY OF SERVICES APPLIED TO A UNIVERSITY LIBRARY

\section{ABSTRACT}

The work has identified four dimensións related to service quality of the Library of the Universidad Nacional Mayor de San Marcos UNMSM. Three of them, which has been called "Tangible Aspects, Empathy and Reliability" can be considered equivalent to those originally obtained by Parasuraman, Zeithaml and Berry. The remaining dimensión is the integrated by Capacity of Response and Security has been called "Response-Security". The dimensións found are consistent with the findings of other research in the university sector, but not included among those found by those authors. Also, has been tested the use of Neural Networks techniques and grouping with $\mathrm{K}$ Means, giving an equivalent result to factorial analysis. In the case of neural networks was used competitive learning with Learning Vector Quantitative LVQ.

Keywords: measuring service quality, library, factorial analysis, $\mathrm{k}$ means, neural networks

\section{INTRODUCCIÓN}

En la actualidad más del $80 \%$ de las actividades económicas de los países; incluido el Perú, son las actividades de servicios. A pesar de ello es poco lo estudiado a la fecha sobre el tema de la medición de la calidad de los servicios; hay un amplio debate al respecto y diversas propuestas sobre cómo hacerla. El objetivo del trabajo aplicado a una biblioteca universitaria es realizar un estudio comparativo de las dos metodologías de medición de la calidad de servicios más aceptadas, esto es las metodologías Service Quality SERVQUAL y Service Performance SERVPERF, y los avances que existen en la actualidad sobre el tema. Estas dos grandes corrientes de medición de la calidad tienen aspectos positivos, pero no son completas, por ello se requiere analizar las metodologías existentes y con base a ellas desarrollar una propuesta complementaria e integradora.

Gronroos [3] y Lewis [7] plantean que se pueden distinguir dos dimensiónes básicas a la hora de hablar de calidad de servicio: calidad técnica y calidad funcional. Desde esta perspectiva, resulta imprescindible prestar atención al diseño del servicio, a los aspectos más tangibles del mismo, lo que implica valorar correctamente lo que los clientes esperan y también a su realización; es decir, la forma en que se ofrece el servicio y muy especialmente la interacción entre el cliente y el personal de contacto de la empresa [11].

Zeithaml et al. [13] definen la calidad de servicio percibida como "el juicio global del cliente acerca de la excelencia o superioridad del servicio" que resulta de la comparación entre las expectativas previas de los usuarios sobre la misma y las percepciones acerca del desempeño del servicio recibido. En 1990 desarrollaron la metodología SERVQUAL que es la más aceptada en la actualidad para medir la calidad de los servicios.

SERVQUAL aplica un cuestionario desarrollado tras un amplio número de entrevistas con directivos y clientes claves de empresas de servicios de mantenimiento, ban$\mathrm{ca}$, tarjetas de crédito y comunicaciones a larga distancia, que contiene 22 pares de ítems que exploran cinco

1 Doctor en Ingeniería, docente de la Facultad de Ingeniería Industrial - UNMSM. Email: jceval@terra.com.pe 
dimensiónes (seguridad, tangibilidad, capacidad de respuesta, fiabilidad y empatía).

Zeithaml et al. [13] sostienen que la calidad del servicio viene definida por la magnitud y sentido de la diferencia entre lo que el cliente espera (expectativa) y lo que obtiene (percepción).

Si la expectativa supera lo percibido, el cliente considerará que la calidad es mala. La metodología que se sigue, luego de la encuesta de expectativas y percepciones; es determinar la diferencia y con ello aplicar el análisis factorial para determinar las dimensiónes. Con el modelo precisado se aplica en diferentes momentos y se puede determinar si se está mejorando o no. También se puede planificar un programa de mejora con base a las diferencias o brechas encontradas.

Teas, K. [9] desarrollaró un estudio complementario a los avances de Zeithaml et al., en el cual critica la propuesta de medir la calidad mediante la diferencia entre percepión y expectativas; especialmente en lo que se refiere a la medición de las expectativas. Al respecto, propociona recomendaciones a tener en cuenta en la determinación de las expectativas. Grady,M; Cronin,J y Brand, R. [2], en su trabajo "Performance - only measurement of service quality: a replication and extension"; hacen un análisis comparativo de las metodologías SERVQUAL y SERVPERF. Habiendo definido SERVPERF como una metodología que hace su análisis sólo con base en las percepciones. Ello concluye que resulta posible definir la calidad de servicio exclusivamente en función de las percepciones sobre el desempeño, sin necesidad de medir las expectativas.

Hong Qin [5] hace una propuesta sobre SERVPERF a los FFR en la China que se desarrolla con base a las 5 dimensiónes de SERVQUAL, pero sólo tomando en cuenta el sistema SERVPERF.

Mira, J., Buil, A., Rodríguez, J. y Aranaz J. [8], después de analizar las diversas propuestas de medicación de la calidad de servicio, llegan a la conclusión de que lo más conveniente es aplicar el sistema SERVQUAL; considerando el concepto de los GAPS (brechas) y sin dejar de considerar el concepto de las expectativas; y luego aplican su propuesta a las ciencias de la salud;

Van Campen, et al. [10] compararon cuatro de los instrumentos de medida de la satisfacción más utilizados (PSQ, CSQ, SPPCS, PJHQ) con SERVQUAL, y recomiendan pese a todo la utilización de este último en base a los datos sobre fundamentación teórica, validez de construcción, validez discriminante, fiabilidad y facilidad de uso. Sin embargo, recomiendan adaptar SERVQUAL a cada medio.

Hughey, H. y Chawla, S. [6] en su trabajo "Measuring the Quality of University Computer Labs Using SERVQUAL: a Longitudinal Study", sostienen que a pesar de las críticas y los paños que han pasado el sistema SERVQUAL se mantiene en uso y se ha aplicado a diferentes tipos de servicios. Al aplicar el análisis factorial se llegó a un modelo con tres factores. Asimismo, aplicaron el método en dos momentos diferentes habiendo obtenido las brechas respectivas en el tiempo transcurrido.

Zamudio, M., Cardoso, M. y Castro, W. [12] en su trabajo "El Uso del SERVQUAL en la Verificación de la Calidad de los Servicios de Unidades de Información: El caso de la Biblioteca del Instituto de Pesquisas Energéticas e Nucleares (IPEN)", utilizaron el modelo del SERVQUAL, que identifica la diferencia entre la expectativa y la satisfacción del usuario con los servicios ofrecidos por la biblioteca. La investigación fue realizada en el segundo semestre de 2003, vía cuestionario electrónico, con 620 usuarios pertenecientes a la comunidad científica del IPEN, de los cuales el $80 \%$ respondieron. A partir del análisis de los resultados se constató que la calidad de los servicios ofrecidos por la biblioteca que está muy cerca de lo considerado ideal por los usuarios, quedan, sin embargo, algunos puntos susceptibles de mejoría.

Fonte, M., Guerrero, G. y Giráldez, R. [1] aplicaron el Modelo SERVQUAL, con procesador estadístico SPSS. Entre los principales resultados sostienen que existe un nivel medio de calidad representado por una media total de 3.10 en una escala de 1 a 5 , esto indica que el cliente externo está recibiendo igual a lo esperado. El 94\% de la población encuestada marca entre igual y más de lo esperado, por lo que este valor se corresponde con el por ciento de la población satisfecha. Este trabajo permitirá la aplicación de un programa de mejoras sobre la base de la Gestión Total de la Calidad.

Justamente por lo antes mencionado en el presente trabajo se tomarán en cuenta los 2 métodos más aceptados SERVQUAL y SERVPERF y se aplicarán a un servicio de biblioteca universitaria.

\section{METODOLOGÍA}

Los principales pasos que se han seguido son 1) Se elaboró la encuesta de 22 ítems tanto para las expectativas como para la percepción; 2) Se determinó el tamaño de muestra; 3) Se aplicó la encuesta por estudiantes del Curso de Sistemas de Calidad de la Facultad de Ingeniería Industrial de la UNMSM (12 en total); 4) Se procesó la informa- 
ción, mediante la técnica del Análisis Factorial y con uso del Software SPSS 18. Asimismo, se aplicó las técnicas de agrupación de datos K Means, Redes Neuronales de Mapas Auto - organizados y Redes Neuronales de Aprendizaje Competitivo LVQ; 5) Discusión de los resultados; y 6) Se presentan las conclusiones.

1. Encuesta. Se elaboró la encuesta de 22 ítems tanto para las expectativas como para la percepción. Las preguntas corresponden a las 5 dimensiónes de SERVQUAL, y corresponden a los mismos temas. Se utilizó una Escala de Likert de 1 al 7 (7 para lo mejor). Se aplicaron a los usuarios de la Biblioteca, a la salida de la misma. Las preguntas 1-4 corresponden a la Dimensión Aspectos Tangibles (equipos de aspecto moderno, instalaciones físicas visualmente atractivas, aspecto pulcro, materiales asociados con el servicio de aspecto visualmente atractivo); las preguntas 5-9 corresponden a la Dimensión Fiabilidad (lo que se promete hacer se cumple, interés sincero por resolver problemas, servicio correcto a la primera, proveen servicios en el tiempo que prometen, registros libres de error); las preguntas 10 a 13 corresponden a la Dimensión Capacidad de Respuesta (dicen a sus usuarios exactamente cuando los servicios serán realizados, servicio rápido, siempre dispuestos a ayudar a sus usuarios, nunca estarán demasiado ocupados para atender a los usuarios); las preguntas 14-17 corresponden a la Dimensión Seguridad (transmiten confianza en los usuarios, usuarios se sienten seguros en sus atenciones, empleados siempre amables con los usuarios, empleados tienen el conocimiento para responder a los requerimientos de los usuarios) y las preguntas 18-22 corresponden a la Dimensión Empatía (a los usuarios se da atención individual, horarios de trabajo convenientes para todos sus usuarios, empleados que dan atención personal, empleados tienen el máximo interés al escuchar a los usuarios, empleados entienden las necesidades específicas de sus usuarios).

2. Tamaño Muestra. Se aplicó la fórmula de muestreo aleatorio simple, caso de población finita:

$$
\mathrm{n}=\frac{p(1-p)}{\left\{\frac{E}{Z_{\frac{\alpha}{2}}}\right\}+\frac{p(1-p)}{N}}
$$

donde: $\mathrm{n}$ es el tamaño de la muestra; $\mathrm{N}$ es el tamaño de la población; $\mathrm{E}$ es el error muestral; ZS es el estadístico normal estándar; $\alpha$ es el nivel de confianza; y $\mathrm{p}$ es la proporción de la población que tiene la característica que se busca medir. En nues- tro caso, $\mathrm{N}=28786$, el nivel de confianza de $95 \%$, el valor de $p=0.5$ y el error muestral de 0.1 . Se obtuvo un tamaño de muestra de 96. Los estudiantes aplicaron las encuestas y se obtuvieron 129 encuestas válidas. Los estudiantes de la Universidad tienen edades entre 16 y 26 años. La fecha de aplicación de la encuesta fue junio de 2011.

3. Se aplicó la encuesta durante el lapso de 15 días, del 01 al 15/06/2011, en especial entre las 10 am y 6 pm a los alumnos que salían de la biblioteca.

4. Se procesó la información, mediante la técnica del Análisis Factorial y con uso del Software SPSS 18 para determinar las dimensiónes. Con la misma información se obtuvieron las dimensiónes usando la Técnica de Agrupación K Means, con el software SPSS 18; se aplicaron las técnicas de Redes Neuronales de Mapas Auto Organizados y de Redes Neuronales de Aprendizaje Competitivo LVQ Learning Vector Quantative, con el software Matlab 2010a.

5. Se hizo Discusión de los resultados, y

6. Se obtuvieron las conclusiones.

\section{RESULTADOS}

\subsection{Análisis de fiabilidad}

Para realizar el análisis de fiabilidad del instrumento SERVQUAL se utilizó el coeficiente Alfa de Cronbach. El valor alcanzado para este análisis fue 0,95 tanto para los resultados de expectativas, como de percepción y la diferencia entre percepción- expectativas. Lo que indica una alta homogeneidad y equivalencia de respuesta a todos los ítems a la vez y para todos los encuestados.

\subsection{Promedios}

Los promedios de los 129 resultados obtenidos por cada ítem (pregunta), en expectativas, percepción y diferencias percepción - expectativas, se muestran en la Tabla 1.

El promedio de las expectativas es 5.77 mientras que el promedio de la percepción es 4.28; lo cual da un promedio de diferencias (Gaps) de 1.49. Es decir, se aprecia una brecha negativa (de insatisfacción) de 1.49 sobre un valor total de 7 . En todos los casos la diferencia es negativa; es decir, en todas las dimensiónes hay insatisfacción.

La dimensión con valor más elevado en las expectativas es Seguridad (ítems 14-17) con 5.901175; mientras que en las percepciones la dimensión con valor más elevado es la Seguridad con puntaje de 4.44765. En lo que respecta a la diferencia P-E más 
Tabla 1. Tabla de Promedios

\begin{tabular}{|c|c|c|c|c|c|}
\hline $\begin{array}{l}\text { Expectativas } \\
\text { İtem }\end{array}$ & Promedio & $\begin{array}{l}\text { Percepción } \\
\text { Ítem }\end{array}$ & Promedio & $\begin{array}{l}\text { Percepción- } \\
\text {-expectativas }\end{array}$ & Promedio \\
\hline 1 & 5.4496 & 1 & 3.7829 & 1 & -1.6667 \\
\hline 2 & 5.3411 & 2 & 4.2636 & 2 & -1.0775 \\
\hline 3 & 5.7752 & 3 & 4.4496 & 3 & -1.3256 \\
\hline 4 & 5.4341 & 4 & 4.2558 & 4 & -1.1783 \\
\hline 5 & 5.6279 & 5 & 4.1628 & 5 & -1.4651 \\
\hline 6 & 6.0000 & 6 & 4.0000 & 6 & -2.0000 \\
\hline 7 & 5.9147 & 7 & 4.3256 & 7 & -1.5891 \\
\hline 8 & 5.8682 & 8 & 4.2558 & 8 & -1.6124 \\
\hline 9 & 5.5504 & 9 & 4.4031 & 9 & -1.1473 \\
\hline 10 & 5.7597 & 10 & 4.3256 & 10 & -1.4341 \\
\hline 11 & 5.9690 & 11 & 4.2713 & 11 & -1.6977 \\
\hline 12 & 5.9690 & 12 & 4.3721 & 12 & -1.5969 \\
\hline 13 & 5.6899 & 13 & 4.1473 & 13 & -1.5426 \\
\hline 14 & 5.7287 & 14 & 4.3643 & 14 & -1.3643 \\
\hline 15 & 5.8915 & 15 & 4.5039 & 15 & -1.3876 \\
\hline 16 & 5.9457 & 16 & 4.5736 & 16 & -1.3721 \\
\hline 17 & 6.0388 & 17 & 4.3488 & 17 & -1.6899 \\
\hline 18 & 5.7829 & 18 & 4.2558 & 18 & -1.5271 \\
\hline 19 & 5.9147 & 19 & 4.5426 & 19 & -1.3721 \\
\hline 20 & 5.6899 & 20 & 4.1860 & 20 & -1.5039 \\
\hline 21 & 5.7519 & 21 & 4.1008 & 21 & -1.6512 \\
\hline 22 & 5.7519 & 22 & 4.3256 & 22 & -1.6512 \\
\hline
\end{tabular}

Fuente: Elaboración propia

grande es la dimensión Capacidad de Respuesta (ítems 10-13) con puntaje de -1.567825 (el mayor GAP). Por el lado de las dimensiónes más bajas en lo que respecta a las Expectativas se tiene los Aspectos Tangibles (ítems 1-4) con 5.5; mientras que en las Percepciones la dimensión más baja es Aspectos Tangibles con 4.187975. En lo que respecta al menor GAP (diferencia entre P-E) es la dimensión Aspectos Tangibles con -1.312025. Es decir, la dimensión con mejor desempeño en la Biblioteca son los Aspectos Tangibles.

\subsection{Promedio en orden descendente}

Los promedios en orden descendente de los 129 resultados obtenidos por cada ítem (pregunta), en expectativas, percepción y diferencias percepciónexpectativas, se muestran en la Tabla 2.

El ítem con mayor puntaje en las Expectativas es el 17 , que se refiere al conocimiento de los empleados, mientras que la menor expectativa es por el ítem 2 referido a que las instalaciones sean visual- mente atractivas. En lo que respecta a la Percepción el mayor valor es el del ítem 16 sobre que los empleados son siempre amables; y el menor puntaje es del ítem 1 referido a que el aspecto no es moderno (con un puntaje de 3.78 de 7 ). El mejor desempeño; es decir, el menor GAP es del ítem 2 referido a que las instalaciones sean visualmente atractivas y el mayor Gap es el del ítem 6 referido a que la biblioteca no muestra un interés sincero por resolver los problemas de sus usuarios.

\subsection{Aplicación del Análisis Factorial}

Análisis Factorial Percepciones-Expectativas: SERVQUAL

Se utilizó el software SPSS 18; y al aplicar la prueba de KMO se obtuvo .920 y en la prueba de esfericidad de Bartlett se obtuvo un nivel de significación de 0.0 lo cual indica que los datos se adecuan para aplicar el Análisis Factorial; con índice de sedimentación mayor de 1 se obtuvieron 3 factores pero varios casos de variables que eran componentes de 
Tabla 2. Promedios en Orden descendente

\begin{tabular}{|c|c|c|c|c|c|}
\hline $\begin{array}{c}\text { Expectativas } \\
\text { İtem }\end{array}$ & Promedio & $\begin{array}{c}\text { Percepción } \\
\text { İtem }\end{array}$ & Promedio & $\begin{array}{l}\text { Percepción - } \\
\text { Expectativas }\end{array}$ & Promedio \\
\hline 17 & 6.0388 & 16 & 4.5736 & 2 & -1.0775 \\
\hline 6 & 6 & 19 & 4.5426 & 9 & -1.1473 \\
\hline 11 & 5.969 & 15 & 4.5039 & 4 & -1.1783 \\
\hline 12 & 5.969 & 3 & 4.4496 & 3 & -1.3256 \\
\hline 16 & 5.9457 & 9 & 4.4031 & 14 & -1.3643 \\
\hline 7 & 5.9147 & 12 & 4.3721 & 16 & -1.3721 \\
\hline 19 & 5.9147 & 14 & 4.3643 & 19 & -1.3721 \\
\hline 15 & 5.8915 & 17 & 4.3488 & 15 & -1.3876 \\
\hline 8 & 5.8682 & 7 & 4.3256 & 10 & -1.4341 \\
\hline 18 & 5.7829 & 10 & 4.3256 & 5 & -1.4651 \\
\hline 3 & 5.7752 & 22 & 4.3256 & 20 & -1.5039 \\
\hline 10 & 5.7597 & 11 & 4.2713 & 18 & -1.5271 \\
\hline 21 & 5.7519 & 2 & 4.2636 & 13 & -1.5426 \\
\hline 22 & 5.7519 & 4 & 4.2558 & 7 & -1.5891 \\
\hline 14 & 5.7287 & 8 & 4.2558 & 12 & -1.5969 \\
\hline 13 & 5.6899 & 18 & 4.2558 & 8 & -1.6124 \\
\hline 20 & 5.6899 & 20 & 4.186 & 21 & -1.6512 \\
\hline 5 & 5.6279 & 5 & 4.1628 & 22 & -1.6512 \\
\hline 9 & 5.5504 & 13 & 4.1473 & 1 & -1.6667 \\
\hline 1 & 5.4496 & 21 & 4.1008 & 17 & -1.6899 \\
\hline 4 & 5.4341 & 6 & 4 & 11 & -1.6977 \\
\hline 2 & 5.3411 & 1 & 3.7829 & 6 & -2 \\
\hline
\end{tabular}

Fuente: Elaboración propia.

más de un factor, por lo que se hizo varias pruebas de rotación y se ajustó el número de variables, llegándose al resultado más adecuado con 4 factores que representan el $62.955 \%$ de la variación; el método de extracción que dio mejor resultado fue la Factorización Alfa y el método de rotación es de Normalización Promax con Kaiser; y el resultado se logró luego e seis iteraciones. Para llegar al óptimo se eliminó las variables 12, 13 y 19; quedando las agrupaciones por factores así: Factor 1: variables 8, 9, 10, 11, 14, 15, 16 y 17 (Dimensiones Capacidad de Respuesta y Seguridad agrupadas por el resultado del análisis factorial); Factor 2: variables 18, 19, 20, 21 y 22 (Dimensión Empatía); Factor 3: variables 1, 2, 3 y 4 (Dimensión Aspectos Tangibles); y Factor 4: variables 5, 6 y 7 (Dimensión Fiabilidad). En la Tabla 3, se presenta el resultado de manera resumida del análisis factorial con SPSS.
Es decir expresado como ecuaciones las relaciones son:

Calidad $=\mathrm{F} 1+\mathrm{F} 2+\mathrm{F} 3+\mathrm{F} 4$

$\mathrm{F} 1=.715 \mathrm{~V} 8+.535 \mathrm{~V} 9+.898 \mathrm{~V} 10+.516 \mathrm{~V} 11+.728$

$\mathrm{V} 14+.826 \mathrm{~V} 15+.535 \mathrm{~V} 16+.546 \mathrm{~V} 17$

$\mathrm{F} 2=.644 \mathrm{~V} 18+.650 \mathrm{~V} 20+.973 \mathrm{~V} 21+.754 \mathrm{~V} 22$

$\mathrm{F} 3=.704 \mathrm{~V} 1+1.016 \mathrm{~V} 2+.602 \mathrm{~V} 3+.450 \mathrm{~V} 4$

$\mathrm{F} 4=.650 \mathrm{~V} 5+.783 \mathrm{~V} 6+.356 \mathrm{~V} 7$

Análisis Factorial Percepciones: SERVPERF

Se utilizó el software SPSS 18; y al aplicar la prueba de KMO se obtuvo .907 y en la prueba de esfericidad de Bartlett se obtuvo un nivel de significación de 0.0 lo cual indica que los datos se adecuan para aplicar el Análisis Factorial; con índice de sedimentación mayor de 1 se obtuvieron 3 factores pero varios casos de variables que eran componentes de 
Tabla 3. Resultados del Análisis Factorial SERVQUAL Percepción - Expectativas

\begin{tabular}{|c|c|c|c|c|c|c|c|c|}
\hline \multicolumn{4}{|c|}{ Extracción } & \multirow{2}{*}{$\begin{array}{l}\text { Ítem } \\
\text { P-E }\end{array}$} & \multicolumn{4}{|c|}{ Factor } \\
\hline Factor & Total & \%varianza & \%acumulado & & 1 & 2 & 3 & 4 \\
\hline 1 & 9.436 & 49.664 & 49.664 & 1 & & & .704 & \\
\hline 2 & 1.378 & 7.253 & 56.917 & 2 & & & 1.016 & \\
\hline 3 & .616 & 3.245 & 60.162 & 3 & & & .602 & \\
\hline \multirow[t]{16}{*}{4} & .531 & 2.793 & 62.955 & 4 & & & .450 & \\
\hline & & & 5 & & & & & .650 \\
\hline & & & 6 & & & & & .783 \\
\hline & & & 7 & & & & & .356 \\
\hline & & & 8 & & & & & \\
\hline & & & 9 & & & & & \\
\hline & & & 10 & & & & & \\
\hline & & & 11 & & & & & \\
\hline & & & 14 & & & & & \\
\hline & & & 15 & & & & & \\
\hline & & & 16 & & & & & \\
\hline & & & 17 & & & & & \\
\hline & & & 18 & & & .644 & & \\
\hline & & & 20 & & & .650 & & \\
\hline & & & 21 & & & .973 & & \\
\hline & & & 22 & & & .754 & & \\
\hline
\end{tabular}

Fuente: Elaboración propia.

más de un factor, por lo que se hizo varias pruebas de rotación y se ajustó el número de variables, llegándose al resultado más adecuado con 4 factores que representan el $69,807 \%$ de la variación; el método de extracción que dio mejor resultado fue la Factorización Alfa y el método de rotación es de Normalización Promax con Kaiser; y el resultado se logró luego de siete iteraciones. Los resultados fueron obtenidos en tabla similar a la de SERVQUAL. Para llegar al óptimo se eliminó las variables 8, 9, 10 y 13; quedando las agrupaciones por factores así: Factor 1: variables 11, 12, 14, 15, 16 y 17 (Dimensiones, Capacidad de Respuesta y Seguridad juntas); Factor 2: variables 18, 19, 20, 21 y 22 (Dimensión Empatía); Factor 3: variables 1, 2, 3 y 4 (Dimensión Aspectos Tangibles); y Factor 4: variables 5, 6 y 7 (Dimensión Fiabilidad).

Es decir expresado como ecuaciones las relaciones son:

Calidad $=\mathrm{F} 1+\mathrm{F} 2+\mathrm{F} 3+\mathrm{F} 4$

$\mathrm{F} 1=.499 \mathrm{~V} 11+.511 \mathrm{~V} 12+.923 \mathrm{~V} 14+.872 \mathrm{~V} 15+.814$

$\mathrm{V} 16+.425 \mathrm{~V} 17$

$\mathrm{F} 2=.703 \mathrm{~V} 18+.657 \mathrm{~V} 19+.974 \mathrm{~V} 20+.701 \mathrm{~V} 21$ +.625 V22
$\mathrm{F} 3=.661 \mathrm{~V} 1+.953 \mathrm{~V} 2+.801 \mathrm{~V} 3+.552 \mathrm{~V} 4$

$\mathrm{F} 4=.752 \mathrm{~V} 5+.864 \mathrm{~V} 6+.739 \vee 7$

3.5 Aplicación de la técnica de Agrupación KMeans, para seleccionar los Factores

Percepción - Expectativas SERVQUAL:

Se utilizó el software SPSS 18; y al aplicar la prueba de K Means se obtuvo resultado con 5 grupos con errores cuadráticos medios que van entre 0 y 2 . La composición de los factores (que corresponden a los grupos o conglomerados) con relación a las variables fue: Factor 1: variables 11, 12, 13, 14, 15, 16, 17, 21 y 22 (Dimensiones Capacidad de Respuesta y Seguridad juntas); Factor 2: variables 1, 2, 3, 4 (Dimensión A. Tangibles); Factor 3: variables 10, 18, 19 y 20 (Dimensión Empatía); Factor 4: variables 5, 6 y 7 (Dimensión Fiabilidad 1) y Factor 5 variables 8 y 9 (Dimensión Fiabilidad 2). En este caso la agrupación es bastante distinta de la formulación de las preguntas. En la Tabla 4 se presentan los resultados.

\section{Percepción SERVPERF:}

Se utilizó el software SPSS 18; y al aplicar la prueba de K Means se obtuvo resultado con 5 Grupos 
con errores cuadráticos medios que van entre 0 y 2 . La composición de los factores (que corresponden a los conglomerados) con relación a las variables fue: Factor 1: variables 1 Y 5 (Dimensiones de A. Tangibles y Fiabilidad); Factor 2: variables 13, 18, 20, 21 y 22 (Dimensión Empatía); Factor 3: variables 2, 3, y 4 (Dimensión A. Tangibles); Factor 4: variables $6,7,8,9,10,11,12,14,15,16$ y 17 (Dimensiones Fiabilidad, Capacidad de Respuesta y Seguridad juntas) y Factor 5 variables 19 (Dimensión Empatía 2). Los resultados fueron obtenidos en tabla similar a la de SERVQUAL. En este caso la agrupación es bastante distinta de la formulación de las preguntas.

\subsection{Aplicación de Redes Neuronales: Mapas Au- to-organizados de Kohonen y Redes de Apren- dizaje Competitivo LVQ Learning Vector Quanti- tative}

\subsubsection{Redes Neuronales con Mapas Auto-orga- nizados}

Percepción - Expectativas: SERVQUAL

Se utilizó el software Matlab 2010 a; se aplicaron Redes Neuronales de tipo Mapas Auto-organizados en dos fases; en la primera fase con cinco neuro- nas se ingreso la matriz $22 \times 129$ para obtener una matriz de $22 \times 5$; y en la segunda fase se ingresó la matriz $22 \times 5$ para agrupar los datos en 5 grupos utilizando nuevamente 5 neuronas; la matriz obtenida se procedió a la simulación con la Red obtenida, se utilizó Simulink obteniendo resultado con 5 grupos con Cuadrado Medio del Error CME menor a 0.1. La composición de los factores (que corresponden a los Grupos) con relación a las variables fue: Factor 1: variable 19 (Dimensión Empatía 1); Factor 2: variables 6 y 12 (Dimensiones Fiabilidad y Capacidad de respuesta); Factor 3: variables 3, 4, 5, 9, 10, 11, 13, 14, 16, 17, 20, 21 (Dimensiones A Tangibles, Fiabilidad, Capacidad de Respuesta y Empatía); Factor 4: variables 1, 2, 7, 8,15 ,18 y 22 (Dimensiones A. Tangibles, Fiabilidad, y Empatía) y Factor 5 sin variables. En este caso la agrupación es bastante distinta de la formulación de las preguntas. En la Tabla 5, se presentan los comandos y resultados obtenidos.

\section{Percepción: SERVPERF}

Se utilizó el software Matlab 2010 a; se aplicaron Redes Neuronales de tipo Mapas Auto Organizados en dos fases; en la primera fase con cinco neuronas se ingreso la matriz $22 \times 129$ para obtener una matriz de 22 x 5; y en la segunda fase se ingresó la

Tabla 4. Resultados del Análisis con K Means para SERVQUAL Percepción - Expectativas

\begin{tabular}{cccccccccccc}
\hline Ítem & Grupo & Dist & Ítem & Gr & Dist & Ítem & Grupo & Dist & Ítem & Grupo & Dist \\
\hline 1 & 2 & 11.945 & 7 & 4 & 11.131 & 13 & 1 & 13.213 & 19 & 3 & 12.930 \\
\hline 2 & 2 & 10.377 & 8 & 5 & 9.721 & 14 & 1 & 12.624 & 20 & 3 & 11.584 \\
\hline 3 & 2 & 11.627 & 9 & 5 & 9.721 & 15 & 1 & 11.866 & 21 & 1 & 11.969 \\
\hline 4 & 2 & 12.029 & 10 & 3 & 12.049 & 16 & 1 & 13.305 & 22 & 1 & 11.677 \\
\hline 5 & 4 & 10.323 & 11 & 1 & 12.024 & 17 & 1 & 12.121 & & \\
\hline 6 & 4 & 11.441 & 12 & 1 & 12.285 & 18 & 3 & 10.662 & & & \\
\hline
\end{tabular}

Fuente: Elaboración propia.

Tabla 5. Resultados del Análisis con Redes Neuronales con Mapas Auto-organizados para SERVQUAL Percepción Expectativas

>> net = newsom(percep1',[5]); wej=net.IW $\{1\}$; net = newsom(percep1',[1]); wej=net.IW $\{1\}$; net

= newsom(wej,[5]); wejj=net.IW\{1\}; s = sim(net,wej); as=vec2ind(s);

Columns 1 through 22

3344334333333334314231

Fuente: Elaboración propia. 
matriz 22 x 5 para agrupar los datos en 5 grupos utilizando nuevamente 5 neuronas; la matriz obtenida se procedió a la simulación con la Red obtenida, se utilizó Simulink obteniendo resultado con 5 grupos con CME menor a 0.1. La composición de los factores (que corresponden a los Grupos) con relación a las variables fue: Factor 1: variable 18 y 22 (Dimensión Empatía 1); Factor 2: variables 20 (Dimensión Empatía 2); Factor 3: variables 1, 2, 5, 6, 8, 9, 10, 11, 12, 13, 14, 15, 17, 21 (Dimensiones A Tangibles, Fiabilidad, Capacidad de Respuesta y Empatía); Factor 4: variables 3, 4, 7, 16,19 (Dimensiones A. Tangibles, Capacidad de Respuesta y Empatía) y Factor 5 sin variables. Los resultados fueron obtenidos en tabla similar a la de SERVQUAL. En este caso la agrupación es bastante distinta de la formulación de las preguntas.

\subsubsection{Redes Neuronales de Aprendizaje Compe- titivo LVQ}

Percepción - Expectativas: SERVQUAL

Se utilizó el software Matlab 2010 a; se aplicaron Redes Neuronales de tipo Aprendizaje Competitivo tipo LVQ; se ingreso la matriz 22 x 129 se trabajó con Red de 5 neuronas para agrupar los datos en 5 grupos; la matriz obtenida se procedió a la simulación con la Red obtenida con un entrenamiento de 5000 epochs, se utilizó Simulink obteniendo resultado con 5 grupos con CME menor a 0.1. La composición de los factores (que corresponden a los Grupos) con relación a las variables fue: Factor 1: variables 1,2,3 y 4 (Dimensión A. Tangibles); Factor 2: variables 5, 6, 7, 11 y 12 (Dimensiones Fiabilidad y Capacidad de Respuesta) ; Factor 3: variables 13, 14, 15, 16 y 17 (Dimensiones Capacidad de Respuesta y Seguridad); Factor 4: variables 8, 9, 21 y 22 (Dimensiones Fiabilidad y Empatía) y Factor 5 variables 10,18,19 y 20 (Dimensiones Capacidad de Respuesta y Empatía). En este caso la agrupación es bastante similar a la formulación de las preguntas. En la Tabla 6 se presentan los comandos y resultados obtenidos.

\section{Percepción: SERVPERF}

Se utilizó el software Matlab 2010 a; se aplicaron Redes Neuronales de tipo Aprendizaje Competitivo tipo LVQ; se ingresó la matriz 22 × 129. Se trabajó con Red de cinco neuronas para agrupar los datos en cinco grupos; la matriz obtenida se procedió a la simulación con la Red obtenida con un entrenamiento de 5000 epochs, se utilizó Simulink obteniendo resultado con cinco grupos con CME menor a 0.1. La composición de los factores (que corresponden a los Grupos) con relación a las variables fue: Factor 1: variables 10, 17, 18 y 19 (Dimensiones Capacidad de Respuesta, Seguridad y Empatía); Factor 2: variables 13, 20, 21 y 22 (Dimensiones Capacidad de Respuesta y Empatía); Factor 3: variables 1, 2, 3, y 4 (Dimensión A. Tangibles); Factor 4: variables 5, 6, 7, 8 y 9 (Dimensión Fiabilidad) y Factor 5 variables 11, 12, 15 y 16 (Dimensiones Capacidad de Respuesta y Seguridad). En este caso la agrupación es bastante distinta a la formulación de las preguntas. Los resultados fueron obtenidos en tabla similar a la de SERVQUAL.

\section{DISCUSIÓN}

En la Tabla 2 de Promedios en orden descendente, se aprecia que en ningún caso la percepción $P$ supera a las expectativas $E$; es decir, todas las diferencias P-E son negativas; siendo la mayor diferencia de todas la del ítem 6 (diferencia de -2); es decir, en la biblioteca muestran poco interés por resolver los problemas de los usuarios. La segunda diferencia negativa, en magnitud, es la del ítem 11; es decir, los usuarios sienten que el servicio no es

Tabla 6. Resultados del Análisis con Redes Neuronales de Aprendizaje Competitivo LVQ para SERVQUAL Percepción - Expectativas

>> net = newc(pes',[5]); wts=net.IW\{1,1\}; net.trainParam.epochs= 5000; net=train(net,pes');
a=sim(net,pes'); ac=vec2ind(a)
Columns 1 through 22
1111222445223333355544

Fuente: Elaboración propia. 
rápido; y la tercera diferencia negativa, en magnitud, es la del ítem 17 que se refiere a que los empleados tienen poco conocimiento para responder a los requerimientos de los usuarios.

En lo que se refiere a las tres expectativas más elevadas de los usuarios se tiene a que los empleados tengan conocimiento para responder a sus requerimientos (ítem 17), a que se muestre un interés sincero en resolver los problemas de los usuarios (ítem 6) y a que se dé un servicio rápido (ítem 11).

En lo que se refiere a la percepción; las tres percepciones más elevadas de los usuarios corresponden a que los empleados siempre son amables (ítem 16), a que se tiene un horario conveniente (ítem 19) y a que los usuarios se sienten seguros cuando son atendidos (ítem 15). Por otro lado, las tres percepciones con puntajes más bajos corresponden a que la biblioteca no tiene equipos de aspecto moderno (ítem 1), a la muestra de poco interés en resolver los problemas de los usuarios (ítem 6) y a que la biblioteca no tiene el máximo interés por escuchar a sus usuarios (ítem 21).

El Análisis Factorial ha permitido obtener cuatro factores, tanto para el caso de Percepciones - Expectativas SERVQUAL como el de Percepciones SERVPERF. En ambos casos se hizo una extracción con la Factorización Alfa y la rotación con el método de Normalización Promax con Kaiser, Aspectos Tangibles F3, Empatía F2, Fiabilidad F4 y Capacidad de Respuesta - Seguridad F1:

1. El Factor F1 en SERVQUAL y en SERVPERF explica el $49 \%$ de la varianza, se podría denominar Rapidez- Seguridad; puesto que recoge aspectos de las preguntas relacionadas con Capacidad de Respuesta y Seguridad. Por lo tanto, integra dos de las cinco dimensiónes inicialmente identificadas por Zeithaml, Parasuramany Berry [13].

2. El Factor $F 2$ en SERVQUAL explica un $7,2 \%$ de la varianza total (en SERVPERF 9,5\%), se podría denominar Empatía; puesto que recoge aspectos de las preguntas relacionadas con la Empatía. Por lo tanto, coincide con una de las cinco dimensiónes inicialmente identificadas por Zeithaml, Parasuramany Berry [13].

3. El Factor F3 en SERVQUAL explica un $3,2 \%$ de la varianza total (en SERVPERF 4,9\%), se podría denominar Aspectos Tangibles; puesto que recoge aspectos de las preguntas relacionadas con los Aspectos Tangibles. Por lo tanto, coincide con una de las cinco dimensiónes inicialmente identificadas por Zeithaml, Parasuramany Berry [13].
4. Finalmente, el Factor F4 en SERVQUAL explica un $2,7 \%$ de la varianza total (en SERVPERF $9,5 \%$ ), se podría denominar Fiabilidad; puesto que recoge aspectos de las preguntas relacionadas con la Fiabilidad. Por lo tanto, coincide con una de las cinco dimensiónes inicialmente identificadas por Zeithaml, Parasuramany Berry [13].

En el caso de la agrupación con la técnica de $\mathrm{K}$ Means se ha obtenido un resultado coincidente con el Análisis Factorial para el caso de SERVQUAL más no así en el caso de SERVPERF. En ambos casos se trabajó con cinco agrupaciones. En el caso de SERVQUAL, se obtuvo resultados de una Dimensión de Capacidad de Respuesta y Seguridad unidas; otra Dimensión de Aspectos Tangibles, otra de Empatía y la Fiabilidad está separada en los dos factores que se podrían integrar en una sola dimensión. Los resultados para SERVPERF no permiten una clara diferenciación de las dimensiónes, por tanto esta técnica no sería recomendable aplicar en este caso.

En el caso de la agrupación con la técnica de Redes Neuronales con Mapas Auto-organizados se ha obtenido un resultado bastante distinto al del Análisis Factorial tanto para el caso de SERVQUAL como SERVPERF. En ambos casos los resultados muestran que no se permite una clara diferenciación de las dimensiónes, por tanto esta técnica no sería recomendable aplicar. En estos casos hay que destacar que las agrupaciones no se dan con base a la varianza sino con base en las distancias euclídeas a centros determinados por el algoritmo respectivo de los Mapas Auto-organizados; prácticamente se trabaja con promedios.

En el caso de la agrupación con la técnica de Redes de Aprendizaje Competitivo LVQ para el caso de SERVQUAL equivalente al Análisis Factorial, porque permite clasificar los resultados en las cinco dimensiónes; pero que si se junta en cuatro, se tendría Aspectos Tangibles, Empatía, Seguridad y la combinación de Fiabilidad con Capacidad de Respuesta. Para el caso de SERVPERF también se tiene un resultado equivalente al Análisis Factorial, porque se permite clasificar los resultados en las 5 dimensiónes; pero que si se junta en cuatro, tendríamos Aspectos Tangibles, Fiabilidad, Seguridad y la combinación de Empatía con Capacidad de Respuesta. En estos casos hay que destacar que las agrupaciones no se dan con base a la varianza, sino con base en las distancias euclídeas que van variando con el ingreso de los datos a centros determinados por el algoritmo respectivo de Aprendizaje competitivo, y se hicieron 5000 iteraciones, 
lo cual el software lo efectúa en aproximadamente 60 segundos.

Comparado con los resultados de otros estudios realizados en instituciones educativas de nivel superior como por ejemplo, el trabajo de Hughey y Chawla [6] en la Universidad Angelo State, llegó a tres factores; el caso de Mira, Rodríguez y Anaz [8] en el Hospital Universitario de la Universidad de Alicante llegó a cuatro factores, en el trabajo de López y Serrano sobre Hoteles en Cantabria, España, se llegó a cuatro factores; en el presente trabajo se ha llegado también a cuatro factores o Dimensiones. En los estudios realizados por Parasuraman, Zeithaml y Berry, se han llegado a cinco factores pero hay que tener presente que se realizan en sectores empresariales muy competitivos y homogéneos, lo cual redunda en mayor uniformidad de los resultados y así se pueden identificar mejor las expectativas, percepciones y diferencias en cada tipo de servicio. En la Tabla 7, se agrupan los resultados con los diversos métodos.

Tabla 7. Resultados con los métodos estudiados

\begin{tabular}{ccccc}
\hline Métodos & $\begin{array}{c}\text { SERVQUAL } \\
\text { N. }{ }^{\circ} \text { de dimensiónes }\end{array}$ & $\begin{array}{c}\text { Adecuación a } \\
\text { preguntas }\end{array}$ & $\begin{array}{c}\text { SERVPERF } \\
\text { N. }{ }^{\circ} \text { de dimensiónes }\end{array}$ & $\begin{array}{c}\text { Adecuación a } \\
\text { preguntas }\end{array}$ \\
\hline Análisis Factorial & 4 & Sí & 4 & Sí \\
\hline K Means & 5 & No & 5 & No \\
\hline RNA Autoorganizadas & 4 & No & 4 & No \\
\hline RNA Aprendizaje LVQ & 4 & Sí & 4 & Sí \\
\hline
\end{tabular}

Fuente: Elaboración propia.

\section{CONCLUSIONES}

1. El trabajo realizado ha permitido identificar 4 dimensiónes relacionadas con la calidad de los servicios en la Biblioteca Central de la UNM$\mathrm{SM}$. Tres de ellas, a las que se ha denominado "Aspectos Tangibles, Empatía y Fiabilidad", pueden considerarse equivalentes a las inicialmente obtenidas por Parasuraman, Zeithaml y Berry. La restante, es la integración de Capacidad de Respuesta y Seguridad que podríamos denominar "Respuesta -Seguridad", si bien resultan coincidentes con las halladas en otras investigaciones realizadas dentro del sector educativo universitario, no se incluyen dentro de las encontradas por dichos autores. En consecuencia, los resultados obtenidos en el presente trabajo proporcionan evidencia empírica adicional a la afirmación de que la calidad de servicio no siempre está integrada por las cinco dimensiónes propuestas por Zeithaml, Parasuraman y Berry [13].

2. Por otra parte, y respecto de las dimensiónes encontradas, señalar que la que tiene mayor peso en la variación en la que se ha denominado "Respuesta-Seguridad "una circunstancia que pone de manifiesto la importancia que para los usuarios encuestados tienen hoy día los aspectos relacionados con la forma en que se presta el servicio frente a aquellos otros meramente tangibles. Se trata de una circunstancia que ha de hacer reflexionar.

3. Se probó el uso de las técnicas de Redes Neuronales y Agrupación con K Means; dando un resultado equivalente al de Análisis Factorial en el caso de las Redes Neuronales con Aprendizaje Competitivo con LVQ.

\section{REFERENCIAS BIBLIOGRÁFICAS}

[1] Fonte, M., Guerrero, G. y Giráldez, R. (2004). "Diagnóstico y evaluación de la calidad de los servicios bibliotecarios de la Universidad de Matanzas "Camilo Cienfuegos". http://www. bibliociencias.cu/gsdl/collect/eventos/index/assoc/HASHbe58.dir/doc.pdf (visitada el 10-052011). Cuba.

[2] Grady, M., Cronin, J. y Brand, R. (2002). "Performance - only measurement of service quality: a replication an d extension". Journal of Business Research 55,17-31.

[3] Gronroos, C. (1982). "Strategic Management and Marketing in the Service Sector". Swedish School of Economics and Business Administration. Helsinki. 
[4] Gronroos, C. (1984). "A Service Quality Model and its Marketing Implications". European Journal of Marketing. Vol. 18, N. ${ }^{\circ} 4$.

[5] Hong Qin (2009). "Service Quality Perceptions in Fast Food Restaurant in China". Uiversity of North Texas. http://www.decisionsciences.org/ Proceedings/DSI2008/docs/182-8380.pdf (visitada el 10-05-2011)

[6] Hughey, H. y Chawla, S. (2003). Measuring the Quality of University Computer Labs Using SERVQUAL: a Longitudinal Study. Journal of Quality Management. 2003, N. ${ }^{\circ} 3$. EEUU.

[7] Lewis, B (1993) Service Quality Measurement. Marketing Intelligence \& Planning. Vol $11, N{ }^{\circ} 4$.

[8] Mira, J., Buil, A., Rodríguez, J. y Aranaz J. (1997). "Calidad percibida del cuidado hospitalario: adaptación del SERVQUAL al hospital público". Hospital Universitario de Alicante. Gaceta Sanitaria11, 176-189. España.
[9] Teas, K. (1993). Expectations, Performance Evaluation and Consumers' Perceptions of Quality; Journal of Marketing. Vol. 57 18-34.

[10] Van Campen, C. et al. (2005) "Quality of care and patient satisfaction a review of measuring instruments". Medical Care Res Rev. 52:109-133.

[11] Weiermair, K. (1997). "Calidad de Servicios y su gestión en las empresas turísticas". Revista Colombiana de Marketing. Papers de Turisme. N. ${ }^{\circ} 20$.

[12] Zamudio, M.; Cardoso, M. y Castro, W. (2005) "EI Uso del SERVQUAL en la Verificación de la Calidad de los Servicios de Unidades de Información: El caso de la Biblioteca del Instituto de Pesquisas Energéticas e Nucleares (IPEN)". Revista Interamericana de Bibliotecología. Vol. $28,{ }^{\circ}{ }^{\circ} 2$.

[13] Zeithaml, V.; Parasuraman, A. y Berry, L. (1990). "Balancnig Customer Perceptions and Expectations". Ed. Free Press. EE.UU. 